\title{
Deviations from a uniform period spacing of gravity modes in a massive star
}

\author{
Pieter Degroote ${ }^{1}$, Conny Aerts ${ }^{1,2}$, Annie Baglin ${ }^{3}$, Andrea Miglio ${ }^{4}$, Maryline Briquet ${ }^{1}$, Arlette Noels ${ }^{4}$, Ewa Niemczura ${ }^{5}$, \\ Josefina Montalban ${ }^{4}$, Steven Bloemen ${ }^{1}$, Raquel Oreiro ${ }^{1}$, Maja Vučković ${ }^{1}$, Kristof Smolders ${ }^{1}$, Michel Auvergne ${ }^{3}$, \\ Frederic Baudin ${ }^{6}$, Claude Catala ${ }^{3} \&$ Eric Michel ${ }^{3}$
}

The life of a star is dominantly determined by the physical processes in the stellar interior. Unfortunately, we still have a poor understanding of how the stellar gas mixes near the stellar core, preventing precise predictions of stellar evolution ${ }^{1}$. The unknown nature of the mixing processes as well as the extent of the central mixed region is particularly problematic for massive stars ${ }^{2}$. Oscillations in stars with masses a few times that of the Sun offer a unique opportunity to disentangle the nature of various mixing processes, through the distinct signature they leave on period spacings in the gravity mode spectrum ${ }^{3}$. Here we report the detection of numerous gravity modes in a young star with a mass of about seven solar masses. The mean period spacing allows us to estimate the extent of the convective core, and the clear periodic deviation from the mean constrains the location of the chemical transition zone to be at about 10 per cent of the radius and rules out a clearcut profile.

The young massive star HD 50230 is poorly studied, but it is known to have spectral type B3V and a visual magnitude of 8.95. HD 50230 is in its central nuclear-burning phase, just like the Sun, transforming hydrogen into helium in its core (the so-called main sequence phase). This important evolutionary phase covers about $90 \%$ of the life of the star, and the detailed internal structure of the star during this phase determines its ultimate fate $e^{1,2}$. This star is at the limiting mass of ending either as core-collapse supernova or as a white dwarf, enriching the interstellar medium with helium and heavy metals in the former case and carbon in the latter. A highquality continuous photometric light curve with 32-s sampling and with a span of 137 days has been obtained for the star by the Convection Rotation and Planetary Transits $\left(\mathrm{CoRoT}^{4}\right)$ satellite. A linear downward trend was removed from the data, after outliers were excluded from the light curve. The duty cycle of the final set of measurements analysed here is $88.6 \%$ and the noise has an amplitude of $1 \mu \mathrm{mag}$ (Fig. 1). The light curve reveals the presence of numerous oscillation modes.

Stellar oscillations have been found to occur at different stages of stellar life, for a range of stellar masses ${ }^{5}$. The best known case is that of the solar oscillations, which are acoustic modes with periods near 5 min (refs 6-9). The seismic interpretation of the detected solar oscillations led to a drastic improvement in the knowledge of the internal structure of the Sun ${ }^{10,11}$. Meanwhile, similar acoustic modes have been detected in various types of distant stars ${ }^{12-14}$. Gravity modes, on the other hand, probe much deeper layers inside stars and in principle allow the study of the core properties of stars far better than acoustic modes. Although such modes have been detected in massive stars similar to HD $50230^{15,16}$, where they have typical periods of half a day to a few days, their seismic potential could not be exploited because the number of detected modes in one star was far too low.

Frequency analysis of HD 50230's CoRoT light curve resulted in a rich frequency spectrum (see Supplementary Information for an outline of the analysis). We detected hundreds of gravity modes with periods roughly in the range of half a day to five days and amplitudes of millimagnitude level, as well as tens of high-frequency acoustic modes with periods in the range of one to ten hours and with

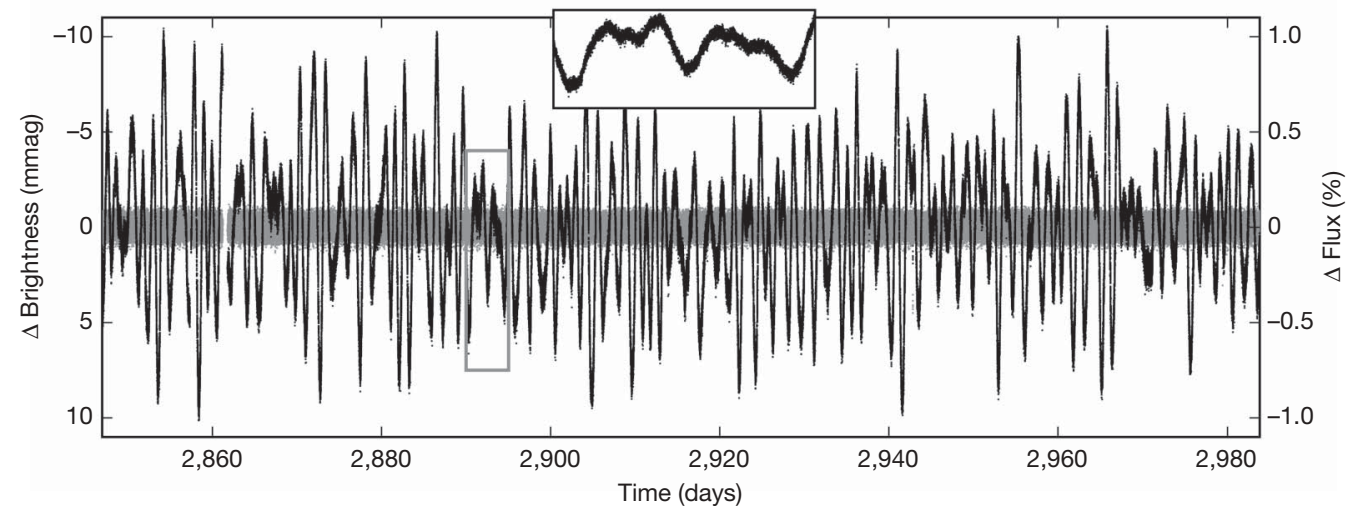

Figure 1 | The light curve of HD 50230 measured by the CoRoT satellite. Main panel, the light curve. Inset, magnified view of the boxed part of the light curve. The residuals resulting from the frequency analysis are plotted in grey.

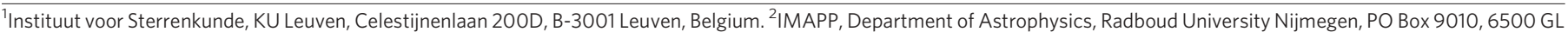

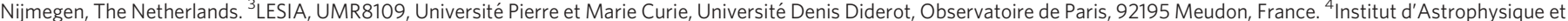

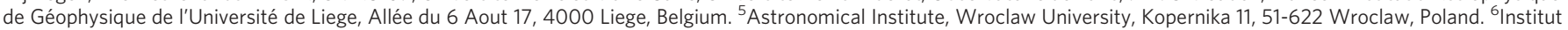
d'Astrophysique Spatiale (IAS), Batiment 121, F-91405, Orsay Cedex, France. 


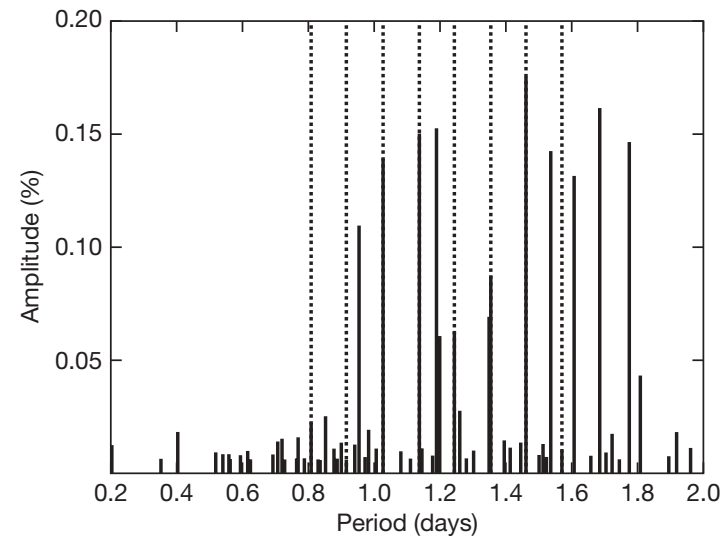

Figure 2 | Extracted frequency spectrum in the g mode region. The vertical dashed lines denote the members of the detected period spacing.

considerably lower amplitudes of several micromagnitudes. This high number of gravity mode frequencies is compatible with our non-adiabatic excitation computations for an appropriate stellar model based on an increase of the metal opacity at temperatures around $200,000 \mathrm{~K}$ (ref. 17). The oscillation period content in the data was extracted using classical and short-time Fourier analysis techniques, combined with nonlinear fitting of the light curve ${ }^{5}$, and a search for a recurrent period spacing was conducted ${ }^{18}$. A group of eight consecutive periods with an almost constant spacing of 9,418 s was detected in the data (Fig. 2). Monte Carlo simulations showed that the probability of a coincidental occurrence of such spacing among eight consecutive oscillation periods within the list of detected frequencies is below $0.1 \%$. Small deviations of up to $200 \mathrm{~s}$ from the constant period spacing occur (Fig. 3). We interpret these as being due to the presence of a region of varying chemical composition outside the convective core ${ }^{3}$. This region is progressively built up during the main sequence by the boundary of the convective core receding towards the centre.

The interior structure of massive stars basically consists of a convective core surrounded by a radiative envelope. With this simplified assumption, theory ${ }^{19}$ predicts the occurrence of a constant period spacing among high-order gravity modes of the same degree. Such a spacing has been found and interpreted previously in white dwarfs, which are stellar remnants at the end of the life of stars like the $\operatorname{Sun}^{20-22}$. They had hitherto not been detected in main-sequence stars. The deviations from the equidistant period spacing carry information on the details of the physical conditions in the stellar interior, which are otherwise inaccessible for observational testing. Observations of binaries and stellar clusters show that a mixed zone larger

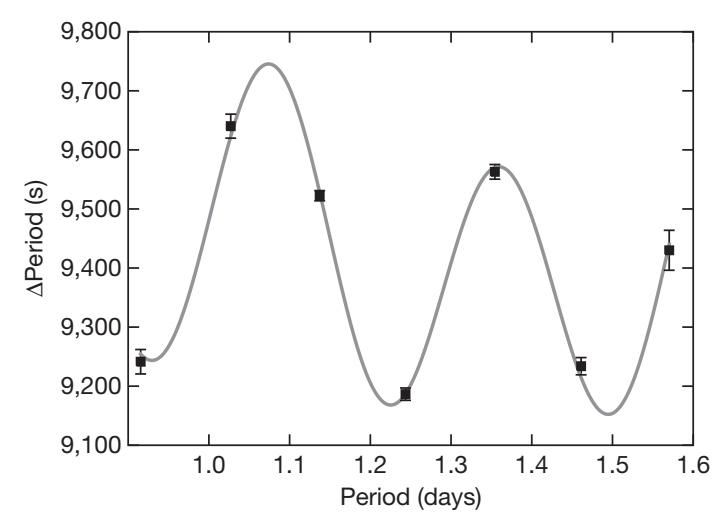

Figure 3 | The components and values of the g mode period spacing. Filled squares, the values of the period components (error bars, s.e.m.). Grey line, a sinusoidal fit with decreasing amplitude, with a period of 2,450 s and maximum amplitude of $240 \mathrm{~s}$. than what is imposed by the convection theory is required ${ }^{23}$. Such an additional mixing could be the result of convective overshooting, that is, the extension of convective motions above the boundary given by the local treatment of convection used in the stellar modelling (the mixing length theory). Other mixing processes include turbulent diffusion or mixing induced by rotation. Theory predicts that the relative importance of these various mixing processes can be separated, as they leave a distinct marker on the spacing structure of gravity modes $^{3}$.

The deviations from the constant period spacing we determined for HD 50230 allow us to evaluate the characteristics of the mixing. To achieve this, the fundamental parameters of the star need to be estimated. For this purpose, several ground-based high-resolution spectra of HD 50230 were recorded with the CORALIE ${ }^{24}$ spectrograph attached to the 1.2-m Euler telescope at the La Silla Observatory in Chile, and with the High Efficiency and Resolution Mercator Echelle Spectrograph (HERMES ${ }^{25}$ ) attached to the $1.2-\mathrm{m}$ Mercator telescope at La Palma. These follow-up spectra revealed a decimal logarithmic gravity of $3.8 \pm 0.2$ (c.g.s. units) and a projected equatorial surface rotational velocity below $5 \mathrm{~km} \mathrm{~s}^{-1}$ from the synthesis of line profiles based on atmosphere models. The effective temperature of the star was estimated to be $17,500 \pm 1,500 \mathrm{~K}$ from its spectral energy distribution, based on archival photometry and space spectroscopy measured with the International Ultraviolet Explorer (see Supplementary Information for a description of the fitting procedure). These results from classical spectroscopy are in full agreement with the spectral type of B3V.

Stellar structure models in accordance with the observed fundamental parameters of HD 50230 were computed with the Code Liégeois d'Évolution Stellaire (CLÉS ${ }^{26}$ ). We considered models without extra mixing, as well as with instantaneous mixing due to core overshooting over a distance parametrized as $0.1-0.3$ local pressure scale heights, or with parametrized turbulent mixing ${ }^{3}$. Under the assumption of solar metallicity and dipolar oscillation modes, the mean spacing of $9,418 \mathrm{~s}$ led us to conclude that the overshoot parameter needs to be at least 0.2 in the radiative regions adjacent to the core to be compatible with the observed effective temperature (Fig. 4). Lowering the metallicity or increasing the degree of the modes increases the amount of extra mixing needed to fit the observed effective temperature.

The observed deviations from constant period spacing (Fig. 3) can be characterized by a periodic behaviour ${ }^{3}$. Its periodicity gives an indication of the evolutionary status in the main-sequence phase. The current observations suggest that the period of the oscillatory part is equal to $2,450 \mathrm{~s}$, indicating that about $60 \%$ of the initial

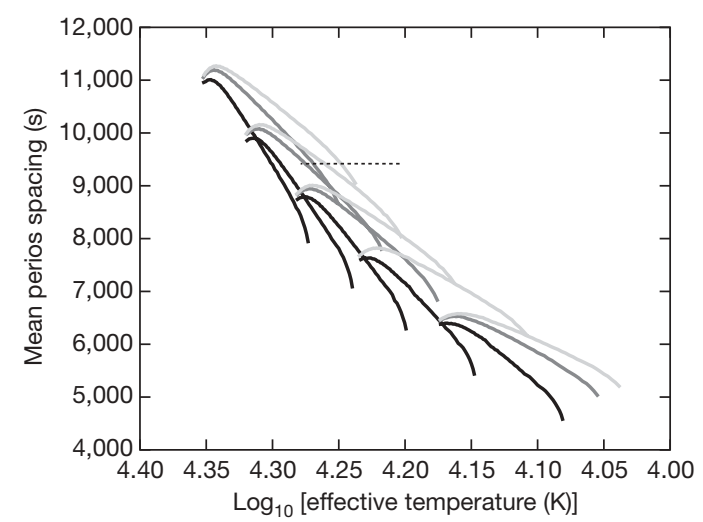

Figure 4 | The influence of the size of the convective mixing region on the mean value of the period spacing. Dashed line, the location of the observed period spacing and the estimate of the effective temperature. Solid lines, the mean period spacing value as a function of the effective temperature, for overshoot parameters of 0 (black), 0.2 (dark grey) and 0.3 (light grey), and different masses (8-4 solar masses from left to right). The metallicity was fixed at $\mathrm{Z}=0.02$. 
hydrogen in the centre of the star has already been consumed. The amplitude of the periodic component is determined by the detailed shape of the chemical composition gradient ${ }^{27}$. The observed small amplitude (240 seconds), decreasing with increasing period, is a clear signature of a smooth gradient of chemical composition. Such a gradient is not compatible with an instantaneous mixing, as the latter would leave such a sharp chemical composition gradient that the amplitude of the periodic components of the deviations from constant period spacing would have a much larger amplitude than that observed-and show no variation with the period.

As a next step, the pressure modes of HD 50230 could in principle be exploited, in addition to the gravity modes discussed here. Without the establishment of a pressure mode frequency spacing, such seismic exploitation of the pressure mode frequencies requires an observational identification of their degree and azimuthal order. This can only be achieved using high-precision multicolour photometric ${ }^{28}$ and high-resolution spectroscopic time series ${ }^{29,30}$, covering a time base similar to that of the CoRoT space photometry.

\section{Received 9 November 2009; accepted 19 January 2010.}

1. Schaller, G., Schaerer, D., Meynet, G. \& Maeder, A. New grids of stellar models from 0.8 to 120 solar masses at $Z=0.020$ and $Z=0.001$. Astron. Astrophys. Suppl. Ser. 96, 269-331 (1992)

2. Maeder, A. Physics, Formation and Evolution of Rotating Stars (Springer, 2009).

3. Miglio, A., Montalban, J., Noels, A. \& Eggenberger, P. Probing the properties of convective cores through g modes: high-order $g$ modes in SPB and Gamma Doradus stars. Mon. Not. R. Astron. Soc. 386, 1487-1502 (2008).

4. Auvergne, M. et al. The CoRoT satellite in flight: description and performance. Astron. Astrophys. 506, 411-424 (2009).

5. Aerts, C., Christensen-Dalsgaard, J. \& Kurtz, D. W. Asteroseismology (Springer, 2009).

6. Leighton, R. B., Noyes, R. W. \& Simon, G. W. Velocity fields in the solar atmosphere. Astrophys. J. 135, 474-499 (1962).

7. Ulrich, R. K. The five-minute oscillations on the solar surface. Astrophys. J. 162, 993-1002 (1970)

8. Leibacher, J. W. \& Stein, R. F. A new description of the solar five-minute oscillation. Astrophys. Lett. 7, 191-192 (1971).

9. Deubner, F. L. Observations of low wavenumber nonradial eigenmodes of the sun. Astron. Astrophys. 44, 371-375 (1975).

10. Christensen-Dalsgaard, J. Helioseismology. Rev. Mod. Phys. 74, 1073-1129 (2002).

11. Chaplin, W. J. \& Basu, S. Perspectives in global helioseismology and the road ahead. Sol. Phys. 1-2, 53-75 (2008).

12. Bedding, T. R. \& Kjeldsen, H. Solar-like oscillations. Publ. Astron. Soc. Aust. 2, 203-212 (2003).

13. De Ridder, J. et al. Non-radial oscillation modes with long lifetimes in giant stars. Nature 459, 398-400 (2009).

14. Michel, E. et al. CoRoT measures solar-like oscillations and granulation in stars hotter than the sun. Science 322, 558-560 (2008).
15. Waelkens, C. Slowly pulsating B stars. Astron. Astrophys. 246, 453-468 (1991).

16. Krisciunas, K. A new class of pulsating stars. Bull. Am. Astron. Soc. 25, 1442-1443 (1993).

17. Dupret, M. A. Nonradial nonadiabatic stellar pulsations. Astron. Astrophys. 366, 166-173 (2001).

18. Degroote, P. et al. CoRoT's view of newly discovered B-star pulsators: results for 358 candidate $B$ pulsators from the initial run's exoplanet field data. Astron. Astrophys. 506, 471-489 (2009).

19. Tassoul, M. Asymptotic approximations for stellar nonradial pulsations. Astrophys. J. Suppl. Ser. 43, 469-490 (1980).

20. Winget, D. E. et al. Asteroseismology of the DOV star PG 1159-035 with the Whole Earth Telescope. Astrophys. J. 378, 326-346 (1991).

21. Winget, D. E. et al. Whole earth telescope observations of the DBV white dwarf GD358. Astrophys. J. 430, 839-849 (1994)

22. Brassard, P., Fontaine, G., Wesemael, F. \& Hansen, C. J. Adiabatic properties of pulsating DA white dwarfs. II - Mode trapping in compositionally stratified models. Astrophys. J. Suppl. Ser. 80, 369-401 (1992).

23. Andersen, J., Clausen, J. V. \& Nordstrom, B. New strong evidence for the importance of convective overshooting in intermediate-mass stars. Astrophys. J. 363, L33-L36 (1990).

24. Baranne, A. et al. ELODIE: a spectrograph for accurate radial velocity measurements. Astron. Astrophys. Suppl. Ser. 119, 373-390 (1996).

25. Raskin, G. \& Van Winckel, H. Hermes, a high-resolution fiber-fed spectrograph for the Mercator Telescope. Proc. SPIE 7014, 178-189 (2008).

26. Scuflaire, R. et al. CLÉS, Code Liégeois d'Évolution Stellaire. Astrophys. Space Sci. 316, 83-91 (2008).

27. Noels, A., Montalban, J., Miglio, A., Godart, M. \& Ventura, P. Overshooting and semiconvection. Astrophys. Space Sci. 326, 233-243 (2009).

28. Aerts, C. et al. Asteroseismology of HD 129929: core overshooting and nonrigid rotation. Science 300, 1926-1928 (2003).

29. Aerts, C. Core overshooting and nonrigid internal rotation of massive stars. Proc. IAU 250, 237-244 (2008).

30. Aerts, C. \& De Cat, P. Beta Cep stars from a spectroscopic point of view. Space Sci. Rev. 105, 453-492 (2003).

Supplementary Information is linked to the online version of the paper at www.nature.com/nature.

Acknowledgements The research leading to these results received funding from the European Research Council under the European Community's Seventh Framework Programme (PROSPERITY), from the Research Council of KU Leuven and from the Belgian Federal Science Policy. The CoRoT space mission has been developed, and is operated by, CNES, with contributions from Austria, Belgium, Brazil, ESA, Germany and Spain.

Author Contributions P.D. and C.A. analysed and interpreted the light curve; M.B., A.M., A.N. and J.M. computed theoretical models; P.D., M.B. and E.N. analysed spectroscopic data; S.B., R.O., M.V. and K.S. made spectroscopic observations; and A.B., M.A., F.B., C.C. and E.M. are the CoRoT instrument builders.

Author Information Reprints and permissions information is available at www.nature.com/reprints. The authors declare no competing financial interests. Correspondence and requests for materials should be addressed to P.D. (pieter.degroote@ster.kuleuven.ac.be). 